\title{
ERICH KÖHLER
}

« Est-ce que l'on sait où l'on va? »Zur strukturellen Einheit von Diderots „Jacques le fataliste et son maître“ 
Sonderdruck

aus

DENIS DIDEROT

Seiten 245-273

\author{
Est-ce que l'on sait où l'on va? \\ Zur strukturellen Einheit \\ von «Jacques le Fataliste et son maître»
}

von

ERICH KÖHLER

WISSENSCHAFTLICHE BUCHGESELLSCHAFT DARMSTADT 


\section{«EST-CE QUE L'ON SAIT OÜ L'ON VA?»- ZUR STRUKTURELLEN EINHEIT VON DIDEROTS «JACQUES LE FATALISTE ET SON MAÎTRE»}

\section{Von ERICH KÖHLER}

Diderots Roman Jacques le Fataliste et son Maître hat es schwer gehabt, als literarisches Kunstwerk Anerkennung zu finden. ${ }^{1}$ Seine philosophische Thematik hat lange Zeit mehr Aufmerksamkeit auf sich gezogen als die Frage nach seiner ästhetischen Struktur, die sich für viele Kritiker überhaupt nicht stellte. Noch heute behaupten P. Castex und P. Surer in ihrem Manuel des études littéraires françaises: «Il [Diderot] est incapable de composer. [. . .] Jacques le Fataliste est un enchevêtrement de récits hétéroclites. ${ }^{2}$ Sie versetzen damit die zahlreichen Benutzer ihres Handbuchs auf den Standort jenes Lesers, dem Diderots Erzähler die aus dem überkommenen Roman bezogenen Erwartungen beharrlich auszutreiben versucht. Zwar hatte sich bereits Goethe von der Charakteristik des Werkes durch den von Diderot eingeführten Leser als einer «insipide rapsodie de faits [...] écrits sans grâce et distribués sans ordre» ${ }^{3}$ nicht täuschen lassen, vielmehr hinter der fingierten Unordnung das System und die kunstvolle Komposition erkannt, ${ }^{4}$ die Diderotfor-

1 Zur Geschichte der Bewertung von Jacques le Fataliste siehe J. Robert Loy, Diderot's Determined Fatalist. A Critical Appreciation of «Jacques le Fataliste» New York 1950, S. 1-24.

${ }^{2}$ Manuel des études littéraires françaises. XVIII' siècle, Paris 1949, S. 97.

3 Diderot, Euvres Romanesques. Texte établi avec une présentation et des notes par Henri Bénac (Classiques Garnier), Paris 1951, S. 714. Nach dieser Ausgabe wird im folgenden zitiert.

${ }^{4}$ Deutlich hebt Goethe in den Anmerkungen zu seiner Übersetzung des Neveu de Rameau seine Meinung von zeitgenössischen Fehlurteilen ab: „Seine Nation, ja sogar seine Freunde, warfen ihm vor, er könne wohl vortreffliche Seiten, aber kein vortreffliches Ganzes schreiben. Diejenigen, die 
schung indessen bedurfte erst der Einsichten der modernen Literaturwissenschaft in die Problematik des Verhältnisses von Autor, Erzähler, Fiktion und Wirklichkeit, um eine ästhetische Würdigung dieses Werks mit Erfolg in Angriff nehmen zu können.

Der unlängst von Paul Vernière erbrachte Nachweis, daß die Genesis von Jacques le Fataliste sich in drei Etappen über einen Zeitraum von fast 29 Jahren erstreckt (von 1765 bis 1784), der Roman mithin ein bis in die letzte „Unordnung" hinein durchdachtes und ausgereiftes Werk darstellen dürfte, ${ }^{5}$ ermächtigt die Forschung, für jedes wie immer willkürlich-zufällig erscheinende Erzählelement die Frage nach seinem Bezug zu den anderen Teilen und zum Ganzen zu stellen, mit anderen Worten: die gemeinsame Ebene freizulegen, auf welcher alle Themen, Motive und Erzählgeschichten konvergieren. Jüngere und jüngste Untersuchungen konnten zeigen, daß eine strukturelle Einheit auch die disparatesten Elemente erfaßt und von ihnen mitkonstituiert wird. Die Inkohärenz des Handlungsablaufs ist selber eines jener Elemente, deren Bedeutung und Funktion nur in ihrer Beziehung zu allen anderen zu erkennen ist, diese Zusammenhänge ganz zu durchschauen, reichen freilich die Mittel einer Literaturwissenschaft nicht aus, die sich der geschichtlichen Perspektive begibt. Alle Versuche, die «organisation cachée» ${ }^{6}$,

so urteilen, hatten wohl den Jacques le Fataliste nicht gelesen; und auch gegenwärtige Schrift gibt ein Zeugnis, wie glücklich er die heterogensten Elemente der Wirklichkeit in ein ideales Ganze zu vereinigen wußte" (Weimarer Ausgabe, Bd. 45, S. 206f.). An anderer Stelle nennt er Jacques le Fataliste eine "sehr köstliche und große Mahlzeit" und bekundet, er habe „in sechs ununterbrochenen Stunden alle Gerichte und Einschiebsel in der Ordnung und nach der Intention dieses köstlichen Kochs und Tafeldeckers verschlungen" (Brief an Merck vom 3. [?] April 1780. Hamburger Ausgabe, Briefe, Bd. 1, S. 300).

5 Paul Vernière, «Diderot et l'invention littéraire: A propos de Jacques le Fataliste» RHLF 59 (1959), S. 153-167. Vgl. dazu jetzt die Ergänzungen und Korrekturen von Jean Varloot, «Jacques le Fataliste et la Correspondance littéraire», RHLF 65 (1965), S. 629-636.

6 Per Nykrog, «Les étapes des Amours de Jacques», in Orbis Litterarum, Supplementum 3 (1963) (Études romanes dédiées à Andréas Blinkenberg), S. 113. 
den «ordre secret» ${ }^{7}$ eines Romans aufzudecken, der 21 eingeschobene Geschichten verschiedenster Art, unterschiedlichen Erzählgrads und jeglichen Umfangs enthält, in dem der Erzählfaden rund $180 \mathrm{mal}$, und das heißt durchschnittlich alle eineinhalb Seiten unterbrochen und neu angeknüpft wird, - alle diese Versuche müssen jedoch selbst dann unbefriedigend bleiben, wenn sich auch eine historisch orientierte Forschung auf die Untersuchung des Fatalismus-Problems und der Struktur des Antiromans beschränkt. ${ }^{8}$ Eine seltsame Scheu, die doch schon durch den Romantitel als wesentlich kenntlich gemachte geschichtliche Dialektik im Verhältnis von Herr und Diener zu erörtern, ist auch in den neueren Interpretationsversuchen festzustellen. Die an Diderot anknüpfende und sein Grundthema philosophisch entfaltende Dialektik von Herr und Knecht in Hegels Pbänomenologie des Geistes ist bisher so wenig wie diejenige des „zerrissenen Bewußtseins“ des Neveu de Rameau für die Diderotforschung fruchtbar gemacht worden. Allein ein Nichtromanist, Hans Mayer, hat wenigstens die Richtung angezeigt, der hier $z u$ folgen wäre. ${ }^{9}$

Die Betrachtung der inzwischen erkannten grundlegenden strukturellen und thematischen Interdependenz von Fatalismusproble-

7 Georges May, «Le maître, la chaîne et le chien dans Jacques le Fataliste», CAIEF 13 (1961), S. 269.

${ }^{8}$ Dies gilt letztlich auch für den interessanten Aufsatz von Roger Laufer, «La structure et la signification de Jacques le Fataliste», RSH 1963, S. 517535. Für Laufer ist es «évident que la signification totale de l'ceuvre ne peut que coïncider avec sa structure» (S. 517). Es ist schade, daß die neuen Ansätze Laufers schließlich in den Versuch einmünden, Jacques le Fataliste als eine «création caractéristique du rococo par'sa structure concentrique» (S. 534) zu erweisen.

9 "Diderot und sein Roman Jacques le Fataliste“ in: Grundpositionen der französischen Aufklärung (Neue Beiträge zur Literaturwissenschaft, Bd. 1), Berlin 1955, S. 55-82. Wiederabgedruckt in H. Mayer, Deutsche Literatur und Weltliteratur, Reden und Aufsätze, Berlin 1957, S. 317-348. - Laufers Anm. 8 genannte Arbeit bleibt auch in dieser Frage in verheißungsvollen Ansätzen stecken. - Rainer Warning weist in seiner scharfsinnigen Studie über "Illusion und Wirklichkeit in Tristram Shandy und Jacques le Fataliste " (Theorie und Geschichte der Literatur und der Schönen Künste, Bd. 4, 
men und Antiromanpolemik ist zu ergänzen durch die Einbeziehung des Themas Herr und Diener, das, wie sich zeigen wird, die eigentliche ästhetische Vermittlungsschicht bildet zwischen dem Unterbau, dem es am nächsten steht, und dem philosophisch-ästhetischen wie auch moralischen Überbau, an dem es in teilweise sogar unmittelbarer Weise partizipiert. Wir fassen die strukturelle Einheit dieser drei thematischen Schichten vorläufig unter dem gemeinsamen Nenner der den Roman einleitenden und in vielfacher Abwandlung leitmotivisch wiederkehrenden Frage: «Est-ce que l'on sait où l'on va?» Sie schließt die absolute Offenheit zukünftigen Geschehens ebenso ein wie dessen Verschlossenheit, und sie ist für das Fatalismusthema genauso betimmend wie für die Problematik des Erzählens und für das Verhältnis von Herr und Knecht. Der Mitvollzug der sich hieraus ergebenden Überlegungen setzt freilich die Bereitschaft voraus, gegebenenfalls auch die These anzuerkennen, daß die bestürzende Modernität der in Jacques le Fataliste ausgetragenen Problematik des Erzählens als Gegenstand des Erzählens selbst, diese Antizipation der Romantheorie des 20. Jahrhunderts, sich letztlich nur aus dem Umstande erklärt, daß Diderot im Durchdenken der gesellschaftlichen Verhältnisse des Ancien Régime bis zum vierten Stand, der im engeren ökonomischen Sinn produktiven Schicht, vorstieß und damit die Grenzen der bürgerlichen Ideologie zu überschreiten vermochte. Daß Diderot den vorrevolutionären Zusammenstoß zwischen Volk und herrschender Schicht in der Auseinandersetzung zwischen Herr und Diener thematisierte, war nichts weniger als ein Zufall: das Leben selbst verwies auf diesen Ort der direkten und permanenten Konfrontation, der zudem in der Komödie längst, im Roman seit Cervantes und dem Picaroroman auch literarisch fixiert war.

Komödie und Roman hatten den Konflikt über die Station der geistigen Überlegenheit des Dieners bis zur Rivalität, zum - freilich vorübergehenden - Rollentausch, ja bis zur Auflehnung gegen eine

München 1965; S. 79) auf die Bedeutung der vorrevolutionären Thematik hin, klammert sie jedoch aus seiner Untersuchung aus, offenbar in der Meinung, sie sei für die ästhetische Struktur irrelevant. 
nunmehr als naturwidrig empfundene Unterdrückung gesteigert. ${ }^{10}$ Aber noch seine entschiedenste Formulierung etwa bei Lesage und Marivaux artikulierte den Anspruch des Dieners in dem Sinne, daß seine Fähigkeiten und seine Leistungen ihn zum Aufstieg wo nicht in die höchsten, so doch in die höheren Schichten legitimieren. Indem der Aufstieg des Dieners bis in den Rang der Noblesse de robe als oberster Grenze geführt und - wie in Marivaux' Paysan parvenu der Aufsteigende sich in virtuoser geistiger und moralischer Anpassung an die höheren Schichten diesen integrierte und damit die Sehnsucht schon des ältesten Picaro der Literaturgeschichte - «arrimarse a los buenos por ser uno dellos ${ }^{11}$ - in vollkommenster Weise erfüllte, war die soziale Dynamik des Konflikts im Sinne der bürgerlichen Ideologie ausgetragen und zugleich aufgefangen. Auch Beaumarchais' Figaro hat diesen ideologischen Bannkreis nicht gesprengt, wie explosiv seine Wirkung am Vorabend der Revolution auch immer gewesen sein mag. Diderot hat auf den literarisch zukunftsreichen Weg zum Entwicklungsroman, der sich für Marivaux aus dieser Konstellation eröffnete, verzichtet und dafür eine neue, revolutionäre Dimension gewonnen, die literarästhetisch, geistesgeschichtlich und literarhistorisch nicht weniger bedeutsam ist als sozialgeschichtlich.

Diderots Jacques strebt weder danach, sich als spekulierendes Finanzgenie einen Platz auf der Sonnenseite des Lebens zu erobern

10 «On a plus d'esprit qu'eux, on les fait vivre, [...] et avec tout cela nous sommes les valets, et ils sont les maîtres. Cela n'est pas juste, $[\ldots]$ je veux devenir maître à mon tour", so gibt der Diener in Regnards Komödie La Sérénade (12. Sz.) seiner Unzufriedenheit Ausdruck (Euvres de J. F. Regnard, Ed. M. Regnard, Garnier, Paris ${ }^{2} 1820$, II. S. 32). Ein Figaros Selbstbewußtsein vorwegnehmendes geistiges Überlegenheitsgefühl offenbart auch Crispin in Regnards Les Folies amoureuses (I, 5). Lesages Crispin Rival de son Maître plakatiert die Rivalität schon im Titel. Der Rollentausch von Herr und Diener ist bis in gefährliche Grenznähe geführt in Marivaux' Ile des Esclaves. Man vergleiche die Empörung des Bauernsohns Jacob in Marivaux' Roman Le Paysan parvenu (Romans, Bibl. de la Pléiade, S. $672 \mathrm{ff}$.).

11 Lazarillo de Tormes, Ed. A. Valbuena Prat, in: La novela picaresca española, Madrid, Aguilar, 1965, S. 85; vgl. auch S. 111. 
wie die valets Crispin und Frontin bei Lesage, ${ }^{12}$ noch hat er den Ehrgeiz, sich einen Adelstitel zu ermogeln und eine amouröse Aufstiegsstrategie zu entwickeln wie der Jacob Marivaux'. Jacques erhebt - anders als der «paysan parvenu ${ }^{13}$ - den Anspruch auf die Anrede «Monsieur» als Jacques, der er bleibt. ${ }^{14}$ Der typische Name für den Bauern individualisiert ihn, während sein Herr namenlos bleibt und sich schon syntaktisch im Romantitel als Attribut seines Dieners erweist. Mit der Umkehrung des alten Sprichworts «tel maître, tel valet» zu «tel valet, tel maître» durch einen Marivauxschen Diener ist hier voller Ernst gemacht. ${ }^{15}$

Was wäre dieser Herr ohne seinen Diener? Der Erzähler läßt uns darüber nicht im Zweifel. Als Jacques sich vorübergehend vom Maître trennt, stellt der Erzähler den Leser vor die Alternative, entweder Jaques oder dem Maître zu folgen. Im letzteren Fall, so bedeutet der Erzähler dem Leser, "vous serez poli, mais très ennuyé; vous ne connaissez pas encore cette espèce-là. Il a peu d'idées dans la tête; s'il lui arrive de dire quelque chose de sensé, c'est de réminiscence ou d'inspiration. Il a des yeux comme vous et moi; mais on ne sait la plupart du temps s'il regarde. Il ne dort pas, il ne veille pas non plus; il se laisse exister: c'est sa fonction habituelle» ${ }^{16}$. «Espèce» ist nach Diderot selbst - im Neveu de Rameau - «de toutes les épithètes la plus redoutable, parce qu'elle marque la médiocrité et le dernier degré du mépris» ${ }^{17}$. Der Neologismus «espèce» verdankt - wie seine Definition durch Duclos erkennen läßt - der sichtbar gewordenen Kluft zwischen dem ständigen Geltungsanspruch und dessen tatsächlicher Erfüllung seine Entstehung. ${ }^{18} \mathrm{Der}$

12 Vgl. Crispin Rival de son Maître, 3. Szene, und Turcaret, V, 14, wo der Diener Frontin zum Schluß nicht ohne Berechtigung behaupten kann: «voilà le règne de $M$. Turcaret fini, le mien va commencer.»

${ }^{13} \mathrm{~S}$. Jacobs Glücksgefühl, nachdem er zum erstenmal in seinem Leben mit «monsieur» angeredet worden ist, a. a. O., S. 722.

14 S. $597 f$.

15 Vgl. Arlequin in La Surprise de l'Amour, II, 5 (Marivaux, Théâtre complet, Bibl. de la Pléiade, S. 170).

16 S. 515.

17 CEuvres Romanesques, a. a. O., S. 474.

18 «L'espèce, terme nouveau, mais qui a un sens juste, est l'opposé de 
Maître verkörpert Langeweile, Inaktivität, Geschehnislosigkeit, was er denkt, stammt nicht von ihm selbst, er ist an sich überflüssig. Nur durch den Diener bewahrt er einen Rest vom Wesen seines Herrentums, das zu bloßen Gewohnheiten erstarrt ist: sein Handeln besteht im Blick auf seine Uhr, im Griff in die Tabaksdose und im Befragen seines Dieners. ${ }^{19}$ Nach der oben zitierten Charakteristik führt der Erzähler die Handlung weiter mit den Worten: «L'automate allait devant lui [...]» Ein Automat funktioniert nur, wenn er bedient wird. Funktionslosigkeit und Unproduktivität bedingen sich - «il se laisse exister; c'est sa fonction habituelle». Die einzige wirkliche Tat, die er vollbringt, die Tötung des Chevalier de SaintOuin, ist unüberlegte, automatische Reflexhandlung, deren Folgen er sich durch Flucht entzieht. Sein Herrentum ist inhaltlose Geste. Was ihm an Wesen bleibt, ist durch den Diener vermittelt; Jacques' Behauptung: «je vous suis essentiel» bleibt unwidersprochen. ${ }^{20}$

So unwesentlich nun der Maître ist, so wesentlich erweist sich die Frage nach dem Maître-Sein. ${ }^{21}$ Nicht für das „einfältige“ Bewußtsein des Maître selbst, für den es so problemlos ist wie alles andere, sondern paradoxerweise für den Diener. «Est-ce qu'on manque jamais de maître dans ce monde?» Jeder Maitre hat seinerseits einen Herrn, so wie sich umgekehrt einen Hund hält, wer sonst niemandem befehlen kann: «chacun a son chien. Le ministre est le chien du roi, le premier commis est le chien du ministre [. . . ] les hommes faibles sont les chiens des hommes fermes» ${ }^{22}$. Ist in dieser Welt, «[où] tout homme voulait commander à un autre homme», das MaîtreSein jemals wirkliches Frei-Sein? Und kann man, wenn Frei-Sein

l'homme de considération: l'espèce est celui qui, n'ayant pas le mérite de son état, se prête encore de lui-même à son avilissement» (Considérations sur les moeurs, 1754). Zit. nach Bénac (Diderot, CEuvres Romanesques, a. a. O., S. 886, Anm. 328).

19 «[...:] il ne savait que devenir sans sa montre, sans sa tabatière et sans Jacques: c'étaient les trois grandes ressources de sa vie [...]» (S. 516). 20 S. 664.

${ }^{21} \mathrm{Zu}$ «maître» als Schlüsselwort des Romans s. G. May in dem oben (Anm. 7) genannten Aufsatz.

${ }^{22}$ S. $667 f$. 
unmöglich ist, wirklich Maître sein? Von seinem Herrn befragt, wozu ihm die versuchte Anpassung an die fatalistische Theorie dienen sollte, antwortet Jacques: «à me rendre parfaitement maître de moi». Aber er fährt fort: «J'y ai renoncé; j'ai pris le parti d'être comme je suis. ${ }^{23}$ Es sieht so aus, als sei die Antwort auf die Frage, weshalb Jacques freiwillig Diener bleibt, angesichts eines solchen Befunds in seinem Bewußtsein darüber zu suchen, daß allein im Dienst für einen Herrn, der in Wahrheit von ihm abhängig ist, sein Diener-Sein aufgehoben ist.

Aber was ist das für ein Diener, der in seine soziale Inferiorität einwilligt wie in ein unabänderliches Schicksal und der sie doch ständig widerlegt! Diderot hat Jacques und den Maître zweifellos im Sinne seiner Theorie von den «conditions» gestaltet. ${ }^{24}$ Diese Theorie ist nicht so $\mathrm{zu}$ verstehen, daß die traditionelle Darstellung von Charakteren einfach durch diejenige der «conditions» ersetzt werden soll. Sie meint vielmehr, daß die Charaktere von der «condition» einerseits und von den Beziehungen, den «rapports» der «conditions» untereinander, und den Konflikten ihrer «intérêts» andererseits bestimmt sein sollen. Diderot sieht darin die Konsequenz einer Orientierung an der gesellschaftlichen Wirklichkeit. Ästhetisch ist damit eine neue Form des Zusammenspiels von Schicksal und Charakter gewonnen. Die persönlichen Konflikte sind Interessenkonflikte, der Charakter ist nur noch die besondere Weise, in welcher die mit Interessenkonflikten beladenen «rapports» der «conditions» individualisiert und mit bestimmten, kollisionsträchtigen Situationen konfrontiert werden: «Le véritable contraste, c'est celui des caractères avec les situations; c'est celui des intérêts avec les intérêts. ${ }^{25}$ Die Analogie von Diderots Ästhetik der «rapports» als der Bedingung des Schönen mit der Theorie der «conditions» bedarf noch eingehender Untersuchung. Für Jacques le Fataliste scheint sie uns evident. Das bedeutet, daß der auf den Fils naturel gemünzte Satz: "Le père aura le caractère de son état»

23 S. 574.

24 Diese Ansicht auch bei Laufer, a. a. O., S. 520.

${ }^{25}$ Diderot, De la poésie dramatique, in Euvres Esthétiques, Ed. Paul Vernière (Classiques Garnier), Paris 1959, S. 234. 
auch auf den Diener Jacques und seinen Maître anzuwenden ist. Das heißt ferner, daß wir die Charakterzüge der beiden Protagonisten auf ihre «conditions» beziehen müssen, daß somit die größere oder geringere Substantialität und Komplexität dieser Charaktere sich von Diderots Begriff ihrer «conditions», vor allem aber von der gegenseitigen Beziehung dieser «conditions», herleitet, die sich zum ganzen Netz ihrer spezifischen Umweltbeziehung weitet und von da her ihren gesellschaftlichen Ort in vollständiger Weise bestimmt. ${ }^{26}$ Die Wahrnehmung dieser «rapports» durch den Dichter aber hat gemäß der Theorie Diderots, dem objektiven Sachverhalt der geschichtlichen Wirklichkeit adäquat zu sein. ${ }^{27}$ Damit aber stellen sich Weltbild und Charaktere der beiden Protagonisten als notwendige individuelle Projektionen ihrer gesellschaftlichen Befindlichkeit dar: die unselbständige, automatenhafte Lebenspraxis des Maître ebenso wie sein folgenloser theoretischer Voluntarismus, die fatalistische Theorie Jacques' nicht weniger als sein dieser entgegengesetztes Handeln. Auch einzelne Charakterzüge des viel profilierteren Dieners sind offensichtlich dialektischer Natur, mit struktureller Ambivalenz ausgestattet: sein «bavardage» ist Zeugnis für die neugewonnene Mündigkeit des Dieners, während Jacques' Großvater noch zum Schweigen verurteilt schien; es ist zugleich eines jener Phänomene, in denen sich die Abhängigkeit des Maitre manifestiert, der ohne des Dieners Redseligkeit der tödlichen Langeweile der eigenen Nichtigkeit verfiele. Gleichwohl wird dem Maître die

26 Vgl. Entretiens sur le Fils naturel, in CEuvres Esthétiques, a. a. O., S. 154: «Nous avons chacun notre état dans la société; mais nous avons affaire à des hommes de tous les états».

${ }^{27}$ Recherches philosophiques sur l'origine et la nature du beau, in CEvres Esthétiques, a. a. O., S. 424: «Quand je dis que Pierre est un bon père je considère en lui une qualité qui suppose l'existence d'une autre, celle de fils; et ainsi des autres rapports, tels qu'ils puissent être. D'où il s'ensuit que, quoique le rapport ne soit que dans notre entendement, quant à la perception, il n'en a pas moins son fondement dans les choses [...]». S. 425: "Quand je dis qu'un être est beau par les rapports qu'on y remarque, je ne parle point des rapports intellectuels ou fictifs que notre imagination y transporte, mais des rapports réels qui y sont, et que notre entendement y remarque par le secours de nos sens.» 
Lust des Zuhörens zuweilen vergällt, denn Jacques hat seine Capricen: das Herrenprivileg der Launenhaftigkeit ist auf den Diener übergegangen.

Diese vom Diener ermöglichte Lust des Maitre am Vernehmen von Geschichten, Surrogat für fehlendes eigenes Tätigsein - oder für ein seltenes Handeln, das nur in Niederlagen endet (s. Liebesgeschichte des Maître) - ist eine originelle Gestaltung dessen, was Hegel den "Genuß" nennt, den der Knecht dem Herrn von dem "Ding" verschafft, das der „Begierde“ des Herrn ohne die Bearbeitung des Knechts nicht zugänglich wäre. ${ }^{28}$ Gerade auch als Erzähler hat Jacques Macht über seinen stoffhungrigen Herrn. In Jacques' Spiel mit der Abhängigkeit des Maitre tritt jene Umkehrung des unselbständigen „knechtischen “ Bewußtseins in ein selbständiges zutage, das sich nach Hegel daraus ergibt, daß der Herr dem Knecht die Bearbeitung des Dings und damit die „Selbständigkeit“ des Dings überlassen hat. So wie der Verlust jeder unmittelbaren Beziehung zum Ding, d. h. jeder echten Produktivität, durch das "Einschieben“ des Knechts „zwischen es und sich“ zum Zwecke des "reinen Genusses“ das "selbständige Bewußtsein“ des Herrn zur Unselbständigkeit umkehrt und damit sein „Wesen“ verkehrt, so wird die Knechtschaft „in ihrer Vollbringung zum Gegenteile dessen werden, was sie unmittelbar ist; sie wird als in sich zurückgedrängtes Bewußtsein in sich gehen und zur wahren Selbständigkeit sich umkehren" 29 . Hegel hat hier als historische Bewußtseinsdialektik formuliert, was er in Diderots Roman erzählerisch gestaltet fand.

Wem in Wahrheit die Verfügbarkeit über die „Dinge“ nunmehr rechtens zusteht, zeigt sich schon deutlich genug in dem Unterschied zwischen der blamablen «histoire des amours du Maittre» und der nur Erfolge verzeichnenden «histoire des amours de Jacques». Was im Gegensatz dieser beiden Geschichten erzählerisch gestaltet wird, findet symbolischen Ausdruck in der Szene des Pferdediebstahls. Der zurückkehrende Jacques findet den Maître schla-

${ }^{28}$ Phänomenologie des Geistes, Ed. Johannes Hoffmeister (Philosophische Bibliothek, Bd. 114), Leipzig 51949, S. 146.

29 Ebda., S. 148. 
fend am Wegrand: seine Hand hält noch den Zügel, aber ein Dieb hat ihn abgeschnitten und das Pferd fortgeführt. ${ }^{30}$ Der Zügel weist den Herrn zwar noch als Besitzer aus, ein anderer aber verfügt über den Besitz. Mit der Antithese von «titre» und «chose» bestimmt Jacques selbst das neue Verhältnis zwischen Herr und Diener einerseits und beider Verhältnis zur Objektwelt andererseits. Nach einem heftigen, durch des Dieners glatte Befehlsverweigerung entstandenen und von der Wirtin vom «Grand-Cerf» zu einem Scheinsieg des Maître geschlichteten Streit, wird Jacques deutlich und entwirft eine Art Vertrag, dessen Artikel - um in der Sprache von Jacques' Fatalismustheorie zu bleiben - die Dekrete des Schicksals formulieren:

Stipulons: $1^{\circ}$ qu'attendu qu'il est écrit là-haut que je vous suis essentiel, et que je sens, que je sais que vous ne pouvez pas vous passer de moi, $j$ 'abuserai de ces avantages toutes et quantes fois que l'occasion s'en présentera. [...]

Stipulons: $2^{\circ}$ qu'attendu qu'il est aussi impossible à Jacques de ne pas connaître son ascendant et sa force sur son maitre, qu’à son maître de méconnaitre sa faiblesse et de se dépouiller de son indulgence, il faut que Jacques soit insolent, et que, pour la paix, son maître ne s'en aperçoive pas. Tout cela s'est arrangé à notre insu, tout cela fut scellé là-haut au moment où la nature fit Jacques et son maître. Il fut arrêté que vous auriez les titres, et que j'aurais la chose. Si vous vouliez vous opposer à la volonté de nature, vous n'y feriez que de l'eau claire. ${ }^{31}$

Die klägliche Argumentation des Maître zugunsten der Willensfreiheit ist längst zusammengebrochen. Auch sein letzter Ausweg, derjenige der Klassenflucht durch Rollentausch mit dem Diener, wird ihm von Jacques versperrt: «Savez-vous ce qui en arriverait? Vous y perdriez le titre, et vous n'auriez pas la chose.»

Die verlorene Macht über das "Ding" ist nicht wiederzugewinnen. Allein das Sichabfinden mit diesem Sachverhalt - vom Maître selbst nun begriffen als «consentement à une loi nécessaire» - beläßt wenigstens noch den «titre». Von seiner einstigen „Selbständigkeit" hat der Herr nur noch den Namen; der Diener bringt ihm

30 S. 520.

31 S. 664f. Hervorhebung vom Verf. 
zum Bewußtsein, «que vous vous appelleriez mon maître, et que c'est moi qui serais le vôtre» ${ }^{32}$. Bald wird diese Behauptung allgemein Geltung haben; insofern glaubt Jacques zu wissen, was der noch nicht lesbare nächste Abschnitt enthält, den das Schicksal auf dem «grand rouleau» verzeichnet hat: «Jacques mène son maître. Nous serons les premiers dont on l'aura dit; mais on le répétera de mille autres qui valent mieux que vous et moi.»

Die Antwort des Maître ist Klage und Resignation in einem: «Cela me semble dur, très dur. ${ }^{33}$ Es bleibt ihm schließlich nichts anderes übrig als zuzugestehen, daß der Diener mit ihm machen kann, was er will, daß in Wahrheit er der Diener und Jacques der Herr ist. ${ }^{34}$ Die Gewißheit seines Überlegenseins verführt Jacques indessen nicht dazu, sich vom Diener in einen Herrn verwandeln zu wollen. Die Fatalismustheorie bewahrt ihn vor einem vorzeitigen Sprung in die Zukunft. Er weiß, daß angesichts der gegebenen gesellschaftlichen Konstellation Herr-Sein auch für ihn den Verlust des „selbständigen Bewußtseins“ und der Verfügung über das „Ding“ bedeuten würde.

Es wäre auch der Verlust des soeben gewonnenen Bewußtseins der Freiheit. Daher erhält der Diener seinem Herrn einen Schein von Selbständigkeit und Herrschaft durch seine Anerkennung als Herrn. Jenes einseitige Anerkennen freilich, in welchem nach $\mathrm{He}-$ gel die erste Stufe des Herr-Knechts-Verhältnisses gründet, ist Vergangenheit. Der Herr hat nicht nur begriffen, daß er Herr nur durch die Anerkennung des Dieners ist, er hat bereits einsehen müssen, daß er seinen Status nur erhalten kann, wenn er die Anerkennung durch den Diener seinerseits als eine solche anerkennt, die nicht mehr von einer Unterlegenheit herkommt, sondern freiwillig und damit widerrufbar ist. Er ist nicht mehr Herr durch Macht,

32 S. 666.

33 S. 665.

34 Der Maître auf die Bemerkung des Marquis des Arcis («vous avez là un serviteur qui n'est pas ordinaire»): «Un serviteur, vous avez bien de la bonté: c'est moi qui suis le sien» (S. 668): «Ce drôle-là fait de moi tout ce qu'il veut ...»(S. 738). Ohne ihn, so bedeutet Jacques, sind die Befehle des Herrn gegenstandslos: «[...] on sait que tous vos ordres ne sont que des clous à soufflet, s'ils n'ont été ratifiés par Jacques» (S. 661). 
sondern durch befristete Zustimmung. Diese gegenseitige Anerkennung, eine Art Vertrag, der indessen nur vom Diener einseitig gekündigt werden kann, beruht auf einer Gleichheit. Auch diese, ja sogar die Möglichkeit einer neuen, anderen Ungleichheit, hat Jacques seinem Maître beibringen müssen. Das Wertbewußtsein seines Herrn hat sich bei dem Gedanken empört, daß die hübsche Denise ihm einen Diener vorzog:

La coquine! préférer un Jacques!

JACQUES

Un Jacques! un Jacques, monsieur, est un homme comme un autre.

Le MaItre

Jacques, tu te trompes, un Jacques n'est point un homme comme un autre. JACQUES

C'est quelquefois mieux qu'un autre. ${ }^{35}$

Vom Maître in seine Schranken gewiesen, erinnert Jacques ihn an das Abenteuer mit den Banditen: «Si, dans la chaumière où nous trouvâmes les coquins, Jacques n'avait pas valu un peu mieux que son maître . . .» ${ }^{36}$ Während der Herr vor Angst zitterte, hatte Jacques ein Dutzend Banditen überrascht und eingeschlossen, mit Hilfe der Pistolen des Maître, der überflüssig gewordenen Attribute des Herrn. ${ }^{37}$

Während Jacques in der Theorie einen uneingeschränkten Determinismus vertritt, handelt er doch selbständig und überlegt. Der Maître dagegen redet von Willensfreiheit und ist unfähig zum Handeln. Sein Bekenntnis zur Freiheit - «Mais il me semble que je sens au dedans de moi-même que je suis libre, comme je sens que je pense ${ }^{38}$ - erfährt durch den Diener eine drastische Widerlegung: Jacques lockert den Sattelgurt am Pferd seines Herrn, der prompt herunterfällt und sich damit nicht bloß zum Testfall für Jacques' Behauptung gemacht sieht, «que nous agissons la plupart du temps sans vouloir», sondern sich auch noch vom Diener sagen lassen muß, daß er dessen Marionette gewesen sei und es nur von Jacques 
abhänge, ob und wie lange er dessen «polichinelle» sei. ${ }^{39}$ Jacques' Erklärung dafür, daß der Maître bei diesem Sturz nicht zu Schaden kam, wirft freilich ein seltsames Licht auf die Schicksalstheorie: «Il était écrit là-haut et dans ma prévoyance que cela n'arriverait pas.» «Ecrit là-haut» ist offenbar, was die Diener tun, und was die Herren erleiden. Der «grand rouleau» hat die tätige Rolle dem Diener reserviert. Was Jacques dem Maître durch diese Demonstration begreiflich machen wollte, ist weniger die Umkehrung der sozialen Verhältnisse, als deren Möglichkeit, die sich indes bereits als wahrscheinlich darstellt.

Vorrangig ist zunächst für das Verhältnis von Herr und Diener die Anerkennung der die Zukunft bestimmenden Frage: «Est-ce que l'on sait où l'on va?» Philosophisch formuliert Jacques diese Frage folgendermaßen: «Est-ce nous qui menons le destin, ou bien est-ce le destin qui nous mène? " ${ }^{40}$ Die Antwort der Protagonisten steht fest: der Maître glaubt an die Macht des freien Willens, das Schicksal zu lenken, für den Diener ist es unbeeinflußbar vorausbestimmt als blindes Fatum. Unabweisbar stellt sich die Frage: was ist die Auffassung Diderots selbst? So sicher es ist, daß die uneingeschränkte Willensfreiheit für Diderot eine absurde Vorstellung war, so ungewiß ist es, ob er einen totalen Determinismus vertrat. Für Jacques le Fataliste ist - wenn auch nicht ohne Vorbehalt - wohl dem Hinweis zu folgen, den Herbert Dieckmann gegeben hat: "Jacques le fataliste ist als Ganzes gesehen nicht, wie so oft behauptet, ein Roman des Determinismus, sondern des sich über ihn erhebenden reflektierenden Bewußtseins und der frei spielenden Einbildungs- und Erfindungskraft. “ 41

Diderot hat sich gehütet, in seinem Roman eine Paradoxie aufzulösen, für die er selber keine Auflösung wußte. Sie ist aber als solche viel mehr in dem widerspruchsvollen und doch geschlossenen Denksystem greifbar, das sich Jacques nach dem Vorbild seines Capitaine gebildet hat, als im Gegensatz der sich ausschließenden Positionen der Willensfreiheit und des Determinismus. Diderots fin-

39 S. 775.

40 S. 504.

41 Herbert Dieckmann, Diderot und Goldoni (Schriften und Vorträge des Petrarca-Instituts Köln, H. 16), Köln 1961, S. 47, Anm. 48. 
gierter Erzähler jedenfalls hält es mit Jacques, wie wir noch sehen werden. Wenn er von seinen beiden Helden sagt: «Ils continuèrent leur route, allant toujours sans savoir où ils allaient, quoiqu'ils sussent à peu près où ils voulaient aller $\aleph^{42}$, so entspricht dies durchaus der Auffassung Jacques', daß der Wille selber ein determiniertes Moment sei, ein Handeln aber deshalb nicht ausschließe. Beides ist zu gleicher Zeit und nebeneinander auf dem "grand rouleau" niedergeschrieben worden. ${ }^{43}$

Die Feststellung: «Jacques disait ce qui était écrit là-haut; son maitre, ce qu'il voulut», kommentiert der Erzähler mit den Worten: «Et ils avaient tous deux raison. $»^{44}$ Es ist kaum zweifelhaft, daß dies die Meinung Diderots ist. Die Kette der determinierenden Ursachen ist so undurchschaubar durchwirkt, daß der Mensch die durch mangelnde Einsicht in die Kausalzusammenhänge entstandenen Lücken mit dem Glauben an die Freiheit seines Handelns ausfüllt. Nicht nur die in Le Rêve de d'Alembert entwickelten Thesen legen diese Konzeption für Jacques le Fataliste nahe, sondern auch die verblüfft zustimmende Reaktion Diderots auf die Definition des Menschen als eines «animal qui se croit libre» durch den Abbé Galiani in einem Brief vom 23. November $1771^{45}$.

Die Möglichkeit, den «grand rouleau» durch Nachdenken zu konsultieren und mittels «prévoyance» und «prudence» dem Destin Schrittmacherdienste zu leisten, ist für Jacques durchaus gegeben. ${ }^{46}$ Wenn Jacques diese Möglichkeit jedoch für die Person des

42 S. 505.

43 Vgl. S. 499: Le Maître: «Je rêve à une chose: c'est si ton bienfaiteur eût été cocu parce qu'il était écrit là-haut; ou si cela était écrit lá-haut parce que tu ferais cocu ton bienfaiteur?» Jacques: «Tous les deux étaient écrits l'un à côté de l'autre. Tout a été écrit à la fois.»

44 S. 513.

45 Brief Galianis an Mme d'Épinay vom 23. Nov. 1771, in: Diderot, Correspondance XI, éd. G. Roth, Paris 1964, S. 225 ff. Auftragsgemäß zeigte Mme d'Épinay den Brief Diderot und berichtet über dessen Reaktion: «J'ai montré votre lettre au philosophe qui en a fait des sauts et des bonds à mourir de rire. Sa perruque n'a jamais autant voyagé sur sa tête que pendant la lecture de cette lettre» (a. a. O., S. 227, Anm. 3).

46 Vgl. S. 543: «Jacques s'arrêta tout court, consulta le destin dans sa tête; il lui sembla que le destin lui disait: Retourne sur tes pas: ce qu'il fit.» 
Herrn bestreitet, so nur deshalb, weil er überzeugt ist, daß die $\mathrm{Zu}-$ kunftswünsche des Maître und der Gang des «destin» unvereinbar sind. ${ }^{47}$ Der Umstand, daß jeder der beiden Protagonisten durch sein tatsächliches Verhalten gleichsam den Beweis für die Richtigkeit der Theorie des andern liefert, rückt damit in eine neue Beleuchtung. Der Widerspruch zwischen Theorie und Praxis bei beiden Figuren - der ja ihren Charakter und ihr Leben wesentlich bestimmt - ist der geschichtlichen Situation immanent. Nicht zufällig ist es Jacques, der Diener, der daraus die Behauptung ableitet: «Un paradoxe n'est pas toujours une fausseté. ${ }^{48}$ Das paradoxale Denken aber, das nach des Maître Meinung im Kopf seines Dieners vorherrscht, hält gerade als Reflex des historischen Widerspruchs seine Aufhebung bereit. Beide, der Widerspruch und seine Aufhebung, sind in Jacques' scheinbar einfältigem Spinozismus geborgen und bewirken die doppelte Struktur seines Fatalismusbegriffs.

Roger Laufer sieht nur eine der beiden wesentlichen Bestimmungen dieses Fatalismusbegriffs, wenn er schreibt: «Le fatalisme est la philosophie de l'homme qui ne peut échapper à sa condition servile: elle lui convient, car elle le justifie. ${ }^{49}$ Laufer trennt die Szenen der Aufsässigkeit des Dieners von dessen Fatalismustheorie, ja er faßt sie, wie dies unausdrücklich fast die gesamte bisherige Forschung tat, als einen inkonsequenten Verstoß gegen die Theorie, nicht weiter reduzierbar als auf den individuellen Charakter Jacques' oder allenfalls auf die Unentschiedenheit Diderots selbst in der Frage des

47 Exemplifiziert in der Diskussion über die Zukunft des Kindes der Agathe, wird aber auch deutlich, wenn der Maître in dem merkwürdigen Verhalten von Jacques' Pferd eine Ankündigung des Schicksals sieht und eindeutig widerlegt wird.

48 S. 545.

49 A. a. O., S. 522. Laufer fährt fort: «C'est ainsi qu'après des moments de dignité où Jacques se révolte, le malaise qui gâte parfois les bons rapports du maître et du valet se dissipe en duplicité et en connivence. Entre la révolte et la soumission du valet, l'abandon et la colère du maître, se situe un terrain de compromis idéologique et moral où règnent la tolérance et l'ironie.» Laufer reduziert den revolutionären Aspekt dieser Theorie auf gelegentliche Revolten des Dieners, die mit dem Fatalismus nichts gemeinsam haben, ja diesem widersprechen. 
Determinismus. Daß diese „Empörung “ in Wahrheit ihre philosophische Begründung in Jacques' Konzeption des Fatums selber hat, läßt sich an der Diskussion demonstrieren, die sich zwischen Herr und Diener über die Zukunft des Kindes der Agathe entspinnt, für das der bös hereingelegte Maître sorgen soll, ohne sein Vater zu sein.

«Qu'il y a d'étranges choses écrites là-haut» - so beginnt Jacques die erbetene Stellungnahme zu den «amours du maître» - «voilà un enfant de fait, Dieu sait comment! Qui sait le rôle que ce petit bâtard jouera dans le monde? Qui sait s'il n'est pas né pour le bonheur ou le bouleversement d'un empire?»

Man kann verstehen, daß der Maître dem Kuckucksei kein Nest aus Daunen bereiten will: «Je te réponds que non. J'en ferai un bon tourneur ou un bon horloger. Il se mariera; il aura des enfants qui tourneront à perpétuité des bâtons de chaise dans ce monde.»

Darauf Jacques: «Oui, si cela est écrit là-haut. Mais pourquoi ne sortirait-il pas un Cromwell de la boutique d'un tourneur? Celui qui fit couper la tête à son roi, n'était-il pas sorti de la boutique d'un brasseur, et ne dit-on pas aujourd'hui? ... .» Der Maître ist am Ende seiner Argumente: «Laissons cela.» ${ }^{50}$

Der Hinweis auf Cromwell einerseits und auf gewisse Gerüchte der Gegenwart andererseits kommt nicht von ungefähr. Die Geschichte hat Jacques recht gegeben. Sein Fatalismus schließt das Sichabfinden mit den für den Augenblick unverrückbaren Zuständen des Ancien Régime, zugleich aber die Gewißheit von deren Veränderung mit ein. $\mathrm{Da}$ Jacques nicht mehr einzusehen vermag, daß sein Knechtsein einer sinnvollen Weltordnung entspricht, ist es für ihn rein zufälliges Ergebnis eines Schicksals, das, unbeeinflußbar durch den menschlichen Willen, morgen alle Verhältnisse umkehren kann. Sein Fatalismus ist durchaus konsequent insofern, als er die Zukunft für völlig offen und unvorhersehbar erklärt und ihr alle Möglichkeiten einräumt außer derjenigen, daß alles so bleibt wie es ist, während der Maître mit der Zuversicht, die Nachkommenschaft Agathes an den Berufsstand des Handwerkers zu fesseln, seine Überzeugung dokumentiert, es genüge der Wille des Herren, den 
gesellschaftlichen Status quo zu erhalten und zu perpetuieren. Jacques' Fatalismus erweist sich demnach als den gegenwärtigen Machtverhältnissen angemessene, vorläufige Hinnahme der bestehenden Gesellschaftsordnung und zugleich als deren Negation, und zwar als eine Negation, die, gerade indem sie sich auf die absolute Kontingenz eines unvorhersehbaren Schicksals gründet, eine optimistische Zukunftsperspektive eröffnet. Von allen Möglichkeiten, die der «grand rouleau » bereithält, ist diejenige, daß sich nichts ändert, die unwahrscheinlichste. Jacques' fatalistische Philosophie birgt die revolutionärste Ideologie, die der Diener angesichts der scheinbar unabänderlichen Zustände ausbilden konnte. Sie gibt Jacques jenes Vertrauen in die Zukunft, aus dem seine Überlegenheit erwächst, das ihm jenen Mut einflößt, der bei vorausgehenden Dienergestalten nur erst als Frechheit - neben obligater Feigheit - sich ankündigte, und das Diderot sogar veranlaßt, ihn durch den Mund des Maître jenen suspekten Philosophen zuzugesellen, die jeden Respekt vor den geheiligten Institutionen der Gesellschaft vermissen lassen. ${ }^{51}$ Der Maître kann trotz seiner freundschaftlichen Gefühle für Jacques nicht verbergen, wie wohl ihm der Gedanke tut, es sei Jacques bestimmt, den Tod der Philosophen, d. h. durch Henkers Hand, zu finden. Das «est-ce que l'on sait où l'on va?» ist für den Herrn jedoch viel bedrohlicher als für den Diener. Das Schicksal ist unabänderlich, was für den Herrn in jeder Hinsicht „fatal“ ist, dem Knecht aber Hoffnung gibt.

Die Umkehrung des Verhältnisses von Herr und Knecht, welche die Negation der bestehenden Gesellschaftsordnung impliziert, führte in der philosophischen Fassung des Fatalismusproblems zur Negation des freien Willens und zur Negation der These von der Bestimmbarkeit der geschichtlichen Zukunft. Mit unvergleichlicher Folgerichtigkeit hat Diderot sie auf die Theorie des Romans übertragen - als Negation des auktorialen Erzählers. Die gesellschaftliche Dialektik wird auf der Ebene der Erzählerproblematik sogar radikaler durchgeführt, weil sie sich dort losgelöst von den unleugbar existenten alten Gesellschaftsformen in gleichsam abstrakter und unangreifbarer Eigendialektik entfalten kann. Die Autonomie 
des Geschehens, logische erzählerische Konsequenz des Fatalismus als Negation der Bestimmbarkeit der geschichtlichen Zukunft, bedeutet die Absage an den traditionellen Roman, dessen Autor selbstherrlich den Weg seiner Figuren bestimmen und über ihr Dasein willkürlich befinden kann. So präsentiert sich Jacques le Fataliste auf jeder Seite als Anti-Roman. Die Feststellung von Diderots Erzähler: «Nous croyons conduire le destin; mais c'est toujours lui qui nous mène ${ }^{52}$, die dasselbe sagt wie der Fatalist Jacques, ist Schritt für Schritt in die Form des Romans umgesetzt und wird dem Leser in unablässiger Apostrophierung eingehämmert. Jeder Augenblick im Romangeschehen hat jene völlig offene, unvorhersehbare Zukunft, die sich aus dem Begriff des kontingenten Schicksals zwangsläufig ergibt. Die Zukunftskategorie der Möglichkeit hat alle sichere Erwartung restlos verdrängt, und nur jene Möglichkeit, auf die der Maître bauen möchte, ist so unwahrscheinlich wie die jeweilige Erwartung des Lesers. Das Naturgesetz kann nicht mehr dazu dienen, den Ewigkeitsanspruch der bestehenden Ordnung zu rechtfertigen, wenn es als kontingentes Schicksal begriffen wird. Das willkürliche Verfügen des Autors über seine Personen ist damit ebenso zu Ende wie das Verfügen des Herrn über den Knecht.

Die Reisefabel mit der ihr innewohnenden Struktur der unberechenbaren Begegnung ist wie keine andere geeignet, die Macht des Zufalls zu demonstrieren, der nun freilich nicht mehr in einer providentiellen Ordnung aufgehoben ist. Das von Diderot entlehnte Aventüren-Schema des Ritterromans (in seiner cervantinischen Parodie) und des Picaroromans erfährt eine radikale Sinnverkehrung. Der Zufall als notwendige Erscheinungsform eines Schicksals, das nicht mehr religiös oder mythisch oder in arrivistische $\mathrm{Zu}-$ versicht eingebettet ist, entspricht jener auch in Jacques lebendigen, in der Literatur des 18. Jh. vielfach belegten Klarheit darüber, daß die Standeszugehörigkeit Resultat eines blinden, sinnfremden $\mathrm{Zu}-$ falls ist..$^{53}$ So ist die Ereignisstruktur von Diderots Roman ihrerseits

\section{S. 520}

53 Man vgl. den berühmten Monolog Figaros in Le Mariage de Figaro. Belege für den «hasard de la naissance» aus dem Werk Holbachs bringt R. Besthorn, Dumarsais-Forschung, in Beiträge zur Romanischen Pbilolo- 
nicht zufällig eine solche der absoluten Kontingenz, die sich noch den Vorgang des Erzählens selber unterwirft und seinen Abschluß in Frage stellt. ${ }^{54}$ Der Zufall war es, der die Begegnung von Jacques mit seinem Maître verursachte. ${ }^{55}$ Der Zufall führt sie am Schluß wieder zusammen, ${ }^{56}$ nachdem der «hasard» der Begegnung mit dem Chevalier de Saint-Ouin sie scheinbar für immer getrennt hatte. ${ }^{57}$ Der Zufall spielt eine wichtige Rolle auch in den eingeschobenen Erzählungen, ${ }^{58}$ vor allem aber beherrscht er die Fabel selbst und durchkreuzt beständig den Vorsatz des Erzählens. Davon werden nicht nur die sekundären Erzähler, wie Jacques, der Maître, oder die Wirtin vom Grand-Cerf betroffen, sondern in erster Linie der den Autor ersetzende Erzähler des Ganzen und mit ihm, ungleich stärker, der Leser.

gie 2 (1963) S. 20. - In Diderots Roman erscheint der «hasard de la naissance» projiziert auf das Moralproblem: «Jacques ne connaissait ni le nom de vice, ni le nom de vertu; il prétendait qu'on était heureusement ou malheureusement né» (S. 670). Die gleiche Erfahrung, erlebt als Diskrepanz zwischen dem Lebensanspruch gut veranlagter Personen und ihrem Schicksal, mit deutlicher Wendung gegen die privilegierten Schichten, findet ihren Niederschlag bereits bei Prévosts Des Grieux: «Ciel! comment traitez-vous avec tant de rigueur le plus parfait de vos ouvrages! Pourquoi ne sommesnous pas nez l'un et l'autre avec des qualitez conformes à notre misère? Nous avons reçu de l'esprit, du goût, des sentimens. Hélas! quel triste usage en faisons-nous? Tandis que tant d'ames basses et dignes de notre sort jouissent de toutes les faveurs de la fortune» (Histoire du Chevalier Des Grieux et de Manon Lescaut. Ed. G. Matoré, Genève-Lille 1953, S. 150).

${ }^{54} \mathrm{Vgl}$. die schon genannten Arbeiten von R. Warning und Georges May, ferner R. Mauzi, La parodie romanesque dans «Jacques le Fataliste», in Diderot Studies 6 (1964), S. $89 \mathrm{ff}$.

55 Der Roman beginnt mit den Sätzen: «Comment s'étaient-ils rencontrés? Par hasard, comme tout le monde» (S. 493).

56 «[Le maître] vivait retiré depuis deux ou trois mois dans le château de Desglands, lorsque le hasard lui rendit un serviteur presque aussi essentiel à son bonheur que sa montre et sa tabatière» ( $\mathrm{S} .780$ ).

57 «L'homme tué était le chevalier de Saint-Ouin, que le hasard avait conduit précisément ce jour-là avec Agathe chez la nourrice de leur enfant» (S. 776).

58 Vgl. S. 583. 
Als Jacques und der Maître einem Reisenden begegnen, der ein Pferd am Zügel führt, wendet sich der Autor an den Leser: «Vous allez croire, lecteur, que ce cheval est celui qu'on a volé au maître de Jacques: et vous vous tromperez. C'est ainsi que cela arriverait dans un roman, un peu plus tôt ou un peu plus tard, de cette manière ou autrement; mais ceci n'est point un roman, je vous l'ai déjà dit, je crois, et je vous le répète encore. ${ }^{59}$ Wie sollte der Leser nach dieser Versicherung damit rechnen können, daß des Maître gestohlenes Pferd sich schließlich doch noch wiederfindet? ${ }^{60}$ Der Autor hält den Leser also in doppelter Weise zum Narren: einmal, um jede vom traditionellen Roman entliehene Erwartung zu enttäuschen, zum anderen, um in potenzierter Weise zu zeigen, daß eben stets das ganz und gar Unerwartete sich einstellen kann. Der Zufall, als Manifestation der Kontingenz, vermag aus der unendlichen Zahl der Möglichkeiten solche beliebig auszuwählen und sie zum völlig unerwarteten Ereignis zu verknüpfen, oder, wie Diderot es in der Erzählung Sur l'Inconséquence du jugement public ... formuliert: «Le hasard combine tous les cas possibles; et il ne lui faut que du temps pour amener la chance fatale. ${ }^{61}$ Indem der Autor das Wiederfinden des Pferdes als unmöglich, weil romanesk hinstellt, um es dann doch stattfinden zu lassen, erhebt er es zu einem jener „bizarren“ Fälle, die der «grand rouleau» für den Menschen bereithält, und die, weil so unberechenbar wie unausweichlich, alles zielgerichtete Planen in Frage stellen: «Ah! monsieur. [...] vous allez voir combien nous sommes peu maitres de nos destinées, et combien il y a de choses bizarres écrites sur le grand rouleau!» ${ }^{62} \mathrm{Die}$ merkwürdige Geschichte von Jacques' Capitaine und dessen Freund benutzt der Autor zu einer grundsätzlichen Erklärung gegenüber dem Leser und seinem Mißtrauen angesichts solcher „außergewöhnlichen " Fälle («deux hommes bien extraordinaires»): «la nature est si variée, surtout dans les instincts et les caractères, qu'il n'y a rien de si bizarre dans l'imagination d'un poète dont
59 S. 528.
60 S. 760.
61 Euvres Romanesques, a. a. O., S. 823.
62 S. 610. 
l'expérience et l'observation ne vous offrissent le modèle dans la nature. $\$ 63$

Mit dem Bizarren als einer vielfältig erfahrenen Tatsache des Lebens selbst stellt Diderot gerade in den eingeschobenen Geschichten die unanfechtbare Wahrheit des Geschehens gegen die künstliche der literarischen Fiktion. Rainer Warning konnte zeigen, wie in diesen Episoden „bizarre zum Inbegriff einer unlösbaren Kasuistik des Lebens, zu einer Kategorie als der objektiven Wirklichkeit “ wird. ${ }^{64}$ Die Ursachen für den von Fritz Schalk verfolgten Bedeutungswandel von «bizarre» von einem negativen $z u$ einem positiven Begriff werden in Diderots Jacques le Fataliste so sichtbar wie sonst

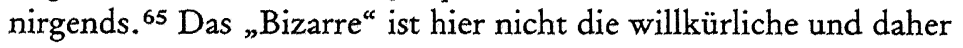
verpönte Abweichung von einer unangefochtenen Norm, ist nicht mehr der eigentlich unzulässige Ausnahmefall, sondern negiert den Geltungsanspruch der Norm in dem Maße, als in ihm ein Wesensgesetz von Natur und Schicksal erkannt wird. Der nicht mehr reduzierbare individuelle Fall widerlegt, allenthalben angetroffen, die seitherige Regel als solche und konstituiert sich selber als ein Gesetz des Lebens. Die gleiche Bizarrerie von Natur und Schicksal ist es, die nunmehr eine Beurteilung von Charakter und Handeln der Individuen gemäß den überkommenen Regeln der Moral ausschließt. ${ }^{66}$ Als „bizarr ${ }^{\text {“ }}$ im negativen Sinne erfährt Jacques jetzt das

63 S. 553.

64 A. a. O., S. 115.

65 F. Schalk, Das Wort „bizarr" im Romanischen, in Etymologica. W. von Wartburg zum 70. Geburtstag, Tübingen 1958, S. 655-679. Diderots Enzyklopädie-Artikel bizarre ist noch an jenen negativen Charakteristika orientiert, die „sehr stark eine im Ancien Régime herrschende Vorstellungswelt erkennen “ lassen, „die sich nach Normen gliedert und der alles Bizarre als etwas Abweichendes erscheint“. Das Bizarre repräsentiert „innerhalb des normativen Denkens die gesetzlose launische Willkür“ (Schalk, a. a. O., S. 668 f.).

66 Dies wird vor allem an den Protagonisten der eingeschobenen Erzählungen demonstriert. Dazu bes. Warning, a. a. O., S. $95 \mathrm{ff}$. - Im „Bizarren“ hat $R$. Warning den Schlüsselbegriff entdeckt, der das Geheimnis der eingeschobenen Geschichten erschließt. Ungeachtet der Tatsache, daß ihnen allen im Rahmen der Haupthandlung und der diese instrumentierenden 
Knecht-Sein, aber es ist für ihn eben doch nur die Bizarrerie des Schicksals, die ihm einen Stand aufzwingt, der im Hinblick auf seine menschlichen Eigenschaften widernatürlich erscheinen muß. Als „bizarr “ empfindet auch Beaumarchais' Figaro den Umstand, daß sein Schicksal von einem Herrn abhängen soll, dem er sich mit

Grundthemen ein bestimmter Stellenwert zukommt, ist ihnen gemeinsam, daß in ihnen das "Bizarre“ "den ästhetischen Reiz eines Verhaltens [bezeichnet], das sich auf keine verbindliche moralische Norm reduzieren läßt", und daß sie „Formen [darstellen], die der Kunstform gegenüber die Position des Nichtliterarischen bezeichnen " wie die selbständigen «contes historiques» Diderots (Warning, a. a. O., S. 105 und 113).

Die Feststellung des Erzählers: «nous croyons conduire le destin, mais c'est toujours lui qui nous mène» (S. 520) gilt auch für die Personen der eingeschobenen Erzählungen, für die Gegner des Abbé Hudson, die dessen Intelligenz und Geschicklichkeit nicht gewachsen sind, ebenso wie für Mme de la Pommeraye, deren raffiniert berechnetes Rachewerk wider alles Erwarten schließlich doch noch scheitert. Es tritt, wie in den Episoden der Haupthandlung, stets das Unerwartete ein, das zuweilen auch das längst aufgegebene Erwartete sein kann, aber nicht mehr als solches zu werten ist. Die Bizarrerie des Geschehens, in der sich außergewöhnliche Charaktere und schicksalhaft-zufällige Ereignisse verbinden können, ist nahezu identisch mit dem Begriff des Unerwarteten, Nicht-Vorhersehbaren, das, indem es die Erwartung der Fiktion als Norm verdrängt, die alte ästhetische Wahrscheinlichkeit außer Geltung setzt. Da die Unvorhersehbarkeit, die Verschlossenheit und Offenheit alles Zukünftigen, zum Prinzip erhoben, das neue Allgemeine, Wesentliche darstellt, ist das mit ihm identische Bizarr„Außergewöhnliche“ nun mit dem Rang des Wahrscheinlichen ausgestattet. Die neue Wahrheit, diejenige, die der Autor „erfindet", um mit ihr kraft einer «illusion du vrai» die Wahrheit der Wirklichkeit zu demonstrieren, ist der „bizarre“ Einzelfall, der sich außerhalb dessen stellt, was bisher als Norm galt. Diese Norm wird daher - ästhetisch und moralisch - als eine historisch bedingte relativiert und als eine überholte abgeschafft. Der Anspruch der alten „Wahrscheinlichkeit ${ }^{\star}$, Ausdruck eines Allgemeinen und damit Notwendigen zu sein, ist erloschen. Hatten im heroisch-galanten Roman nur Personen der Hocharistokratie außergewöhnliche - „bizarre“ Schicksale, deren Singularität nur eine Ausnahmestellung bestätigte, so hat die Vorstellung von der Unberechenbarkeit der Zukunft jetzt den gesamten gesellschaftlichen Raum erfaßt. 
Recht in jeder Hinsicht überlegen weiß. ${ }^{67}$ Für Jacques enthält indessen gerade der „bizarre“ Charakter seines Schicksals bereits auch die Chance für dessen Umkehrung. Die Wiederholung eines so auBergewöhnlichen Falls wie die Erscheinung Cromwells wird auf Grund seiner „bizarren“ Natur ebenso möglich wie der Zufall der Auffindung des gestohlenen Pferdes.

So hat der Begriff des Bizarren aus den gleichen geschichtlichen Gründen dieselbe doppelte Struktur wie der Fatalismusbegriff. Wie dieser enthält er, noch unentbunden, eine Ahnung vom dialektischen Prozeß der Geschichte. Entschiedener noch als im Falle des Fatalismus bezieht hier der Autor Diderot, bzw. sein Erzähler, die Position Jacques'. Was sich als Bizarres, Außergewöhnliches, Vereinzeltes gegen die Norm stellte, nämlich der unzulässige Widerspruch $\mathrm{zu}$ ihr, versetzt, als ein allgemeines Gesetz des Lebens erkannt, die Norm ins Unrecht. Damit wird ästhetisch wahrscheinlich, weil natürlich, was bisher als unwahrscheinlich, weil unnatürlich galt. Diese Umkehrung wird im Dialog zwischen Erzähler und Leser thematisch, und sie bestimmt die Struktur der Fabel von Diderots Roman. Das Bizarre hat mit dem Zufall, der es als Erscheinungsweise der Notwendigkeit im Sinne der Kontingenz des Lebens produziert, die alogische Struktur gemeinsam und stellt sich somit radikal gegen die Logik der alten Romanfabel. ${ }^{68}$ Die Wahrheit des erfahrenen Lebens widerlegt die konstruierte „Wahrheit“ der literarischen Fiktion, die vorgibt zu wissen, wie alles verlaufen muß oder doch müßte. ${ }^{69}$ Was im bisherigen Roman „wahrscheinlich“ war,

67 «Est-il rien de plus bizarre que ma destinée!», Le Mariage de Figaro, V. 3 (Beaumarchais, Théâtre ... Bibl. de la Pléiade, S. 345).

$68 \mathrm{Vgl}$. dazu die scharfsinnigen Darlegungen Warnings, a. a. O., S. $80 \mathrm{ff}$.

${ }^{69}$ Es wäre jedoch u. E. falsch, in dieser Umkehrung der ästhetischen Begriffe von Wahrscheinlichkeit und Notwendigkeit einen Bruch mit der aristotelischen Ästhetik zu sehen, es sei denn, man halte für genuin aristotelisch, was die klassische Doktrin darunter verstand. Die ästhetischen Kategorien des Wahrscheinlichen und des Notwendigen in ihrem spezifischen Verhältnis zum Wirklichen verlieren dadurch, daß ein verändertes geschichtliches Bewußtsein ihre Inhalte anders sieht, nichts von ihrer Gültigkeit. R. Warning hat gewiß recht, wenn er sagt, daß der aristotelische Begriff des Wahrscheinlichen ,unlösbar verknüpft ist mit dem Begriff des 
erweist sich jetzt als „unwahrscheinlich“, während das bislang „Unwahrscheinliche", das, war es überhaupt akzeptierbar, einer umfassenden Begründung als eines im providentiellen Zusammenhang "Notwendigen " bedurfte, $^{70}$ jetzt als einfache Lebenswahrheit ästhetisch zum „Wahrscheinlichen “ wird.

Die Bizarrerie des Lebens darstellen heißt für Diderot «dire la chose comme elle est» ${ }^{71} \mathrm{bzw}$. «comme elle s'est passée» ${ }^{72}$. Das bedeutet auch eine langwierige Umerziehung des Publikums. Der bereits mit den ersten Zeilen einsetzende Dialog zwischen Erzähler

Mythos" und daher ,in dem Moment problematisch werden muß, wo an die Stelle des mythischen ein geschichtliches Geschehen gesetzt wird“ (a. a. O., S. 70). Der Mythos teilt jedoch mit dem Begriff eines geschichtlichen Geschehens, der nicht faktizistisch orientiert ist, die Vorstellung einer allgemeinen Gesetzlichkeit, und gerade diese letztere ist in Jacques le Fataliste nachzuweisen.

70 Ein gutes Beispiel liefert der Streit um die ästhetische Berechtigung des «aveu» in der Princesse de Clèves. Vgl. dazu E. Köhler, Madame de Lafayettes "La Princesse de Clève». Studien zur Form des klassischen Romans (Hamburger Romanistische Studien, Reihe A, Bd. 43), Hamburg 1959, S. $51 \mathrm{ff}$.

${ }^{71}$ Zwar ist es der Maître, der diese Aufforderung an Jacques richtet (S. 544), das «dire la chose comme elle est» ist für ihn jedoch - im Gegensatz zu Jacques - völlig unproblematisch.

72 S. 649. Jacques ist sich klar darüber, wie schwierig es ist, dieser Aufforderung Folge zu leisten, da jeder Sachverhalt nur in der subjektiven Brechung von Erzähler und Zuhörer gemäß ihrer jeweiligen Verfassung ermittelt werden kann: «Cela n'est pas aisé. N'a-t-on pas son caractère, son intérêt, son goût, ses passions, d'après quoi l'on exagère ou l'on atténue? Dis la chose comme elle est! . . Cela n'arrive peut-être pas deux fois en un jour dans une grande ville. Et celui qui vous écoute est-il mieux disposé que celui qui parle? Non. D'où il doit arriver que deux fois à peine en un jour, dans toute une grande ville, on soit entendu comme on dit.» (S. 544). Was für Erzähler und Zuhörer schon schwierig ist, ist es noch mehr für Autor und Leser. Diderot hat diese Schwierigkeit im Nachwort zu den Deux amis de Bourbonne theoretisch zu lösen versucht, praktisch in Ceci n'est pas un conte mit der Einführung des Lesers als eines Zuhörers, der zugleich «personnage» der erzählten Geschichte war und somit aus dem Wissen des Mitbeteiligten heraus den Erzähler korrigieren kann. 
und Leser dient dazu, die Kritik am Roman im Innern des Romans selbst vorzunehmen. Diese Kritik bietet sich wie eine Art Exorzismus dar, der den schier unausrottbaren romanesken Leitbildern des Lesers gilt. Immer, wenn die Handlung einen Punkt erreicht, von dem aus sie im Sinne der traditionellen Fiktion weitergesponnen werden kann, zerschlägt der Erzähler das Erwartungscliché des Lesers im Namen der Lebenswirklichkeit. Die Thematisierung der Diskrepanz von Leben und literarischer Fiktion in Handlung und Kommentar entlarvt unablässig die künstliche Logik der Gattung Roman als Verfälschung der Wirklichkeit. ${ }^{73}$ Das gewohnte Vertrauen des Lesers, vom Autor sicher geführt zu werden und an seinem Vorauswissen teilzuhaben, wird Zug um Zug erschüttert, sein Anspruch darauf für irreal erklärt: «Mais, pour Dieu, l'auteur, me dites-vous, où allaient-ils? . . Mais, pour Dieu, lecteur, vous répondrai-je, est-ce qu'on sait où l'on va?» ${ }^{74}$ Der Autor weiß selber nicht, wohin seine Personen ihn führen werden. Der Vorwurf, den er seinerseits dem Leser macht: «Lecteur, vous me traitez comme un automate ${ }^{75}$, bezieht sich auf jene vorgängige Harmoniegewißheit des fiktionsgewohnten Lesers, deren tröstliche Bestätigung dieser vom Autor erwartet und die dem Glauben des Maître an die Bestimmbarkeit der Zukunft entspricht. In der Tat deutet nicht nur die Verwendung des Wortes «automate» darauf hin, daß der Leser und der Maître zusammengehören. ${ }^{76} \mathrm{Häufig} \mathrm{behandelt} \mathrm{Jacques} \mathrm{sei-}$ nen Herrn wie der Erzähler seinen Leser, ${ }^{77}$ denn sowohl der Leser

73 Vgl. neben zahlreichen anderen Stellen S. 730f., wo der Erzähler alle Möglichkeiten benennt, die ein Romanautor an dieser Stelle nützen könnte, um dann zu betonen: «Je fais l'histoire, cette histoire intéressera ou n'intéressera pas: c'est le moindre de mes soucis. Mon projet est d'être vrai, je l'ai rempli.»

${ }^{74}$ S. 537.

75 S. 556.

76 So wie andererseits der Erzähler und Jacques: Jacques haßt die vom Maître geliebten Portraits, weil sie einen Sachverhalt vortäuschen, welcher der Lebenswirklichkeit niemals standhält. Jacques - hierin Sprecher Diderots - verlangt «faits», "propos», «gestes» anstelle von Portraits (S. $751 \mathrm{f}$.). Vgl. auch die Polemik gegen die Briefe, S. 738.

$77 \mathrm{Zu}$ dieser Frage R. Laufer, a. a. O., S. $530 \mathrm{ff}$. 
wie der Maître glauben zu wissen, wie es weitergeht, weil sie zu wissen glauben, wie es weitergehen sollte. Der Leser, der den Autor auf den weiteren Verlauf der Handlung festlegen möchte, muß die gleiche Zurückweisung hinnehmen wie der neugierige Maître als Zuhörer durch Jacques. Beide werden auf die Gegenwart des jeweiligen Geschehens verwiesen, weil es immer anders kommt, als man annimmt.

Dieses Axiom von Jacques' Fatalismustheorie ist also auch dasjenige des Erzählers: die Autonomie des Geschehens schließt jeden Vorgriff auf die Zukunft aus. Aus diesem Grund werden Erzählzeit und erzählte Zeit vermischt und auf eine Ebene gebracht, welche die Zuhörer aller Grade in unmittelbar Miterlebende verwandelt, denen die Zukunft ebenso verschlossen ist wie den Personen der jeweiligen Erzählung. ${ }^{78}$ Jene im Nachwort zu den Deux Amis de Bourbonne postulierte «illusion du vrai», welche die Grenze zwischen Fiktion und Realität und damit die epische Distanz aufhebt, kann nur zustande kommen, wenn der Autor sich seiner Allwissenheit begibt, d. h. wenn er sich, wie seine «personnages», der Autonomie des Geschehens unterwirft und sich, wie sie und seinen Leser, selber in die jeweilige erzählte Zeit stellt. So wird er in die Lage versetzt, die Stunden, in der seine Helden schlafen, mit dem Erzählen einer Geschichte zu überbrücken ${ }^{79}$ oder sich gar mit seinen Personen zu unterhalten. ${ }^{80}$ Damit wird aber auch fraglich, ob er, nun selber der Kontingenz unterworfen, seine Geschichte

78 Besonders eindrucksvolles Beispiel S. 610: Jacques erzählt von einem erneuten Duell seines Capitaine mit dessen Freund: «Son adversaire envoie à son secours, se met à table avec ses amis et le reste de la carrossée, boit et mange gaiement. Les uns se disposaient à suivre leur route, et les autres à retourner dans la capitale, en masque et sur des chevaux de poste, lorsque l'hôtesse reparut et mit fin au récit de Jacques.

La voilà remontée, et je vous préviens, lecteur, qu'il n'est plus en mon pouvoir de la renvoyer». Vgl. auch S. $599 \mathrm{f}$.

79 S. 583.

80 S. 729 wendet sich der Erzähler an den Leser, um ihm ein weiteres Beispiel zu einem vom Maitre erzählten Ereignis zu berichten, worauf ihm jedoch nicht der Leser, sondern der Maître, und nach diesem Jacques Antwort gibt. 
überhaupt zu Ende zu führen vermag. Den Zweifel hierüber hat er wiederum mit Jacques gemeinsam, der nach wiederholten Unterbrechungen durch die "fatalen" Widerfahrnisse der Reise nicht mehr glaubt, die «histoire de ses amours» zum Abschluß bringen zu können. . $^{81}$

Unablässig wird im Dialog mit dem Leser die Gestalt des traditionellen, willkürlich seine Figuren führenden Autors beschworen «il ne tiendrait qu'à moi [...]», «n'aurais-je pas été le maître de $[\ldots]{ }^{82}-$ um sogleich destruiert zu werden. Der ideal-romaneske Handlungsverlauf ist stets präsent als einer, der vom Leben widerlegt wird, so wie das Herr-Diener-Verhältnis alter Ordnung immer wieder in Erinnerung gebracht wird, um seine Umkehrung zu erfahren. Die Frage, die im Verhältnis von Herr und Diener als eine sozialgeschichtliche gestellt ist, die in der Diskussion über Willensfreiheit und Determinismus philosophisch ausgetragen wird, lautet im Bereich der Erzähltheorie gleich und erzählt dieselbe negative Antwort: kann man das Leben wirklich führen? Der Autor weiß von vorneherein von dem, wovon der Maittre und der Leser erst überzeugt werden müssen: von der Offenheit der Zukunft und ihren unvorhersehbaren Möglichkeiten. Er erfindet Figuren, stellt sie in eine bestimmte Ausgangssituation und setzt sie dem Leben aus. In diesem Augenblick ist seine Macht auch bereits zu Ende: das Leben selbst nimmt die Leitfäden des Marionettenspielers in die Hand. Die Autonomie des Geschehens als «vérité de l'histoire» schließt die Autonomie des Erzählens aus. Der Kommentar, sonst allgemein Kennzeichen der auktorialen Erzählhaltung, vollzieht hier deren Liquidation. Der Autor hat es aufgegeben, das Schicksal $\mathrm{zu}$ gestalten; an seine Stelle tritt das Schicksal selbst, das sich Schritt für Schritt offenbart und das er nur noch Schritt für Schritt begleiten kann. Wo seine Gewährsleute versagen, verzichtet er auf eigenmächtiges Ausfüllen der Lücke. Wenn seine Quelle drei verschiedene Versionen über das Ende der «histoire des amours de

81 Vgl. S. 536, 673, 736 und S. 777: «Et les amours de Jacques? Jacques a dit cent fois qu'il était écrit là-haut qu'il n'en finirait pas l'histoire, et je vois que Jacques avait raison.»

82 S. 526, 551, 731. 
Jacques» bringt, so muß er offenlassen, welche die Wahrheit enthält. ${ }^{83}$

Soll das kontingente Schicksal selber die Handlung führen, so muß der Autor sich selber als Schöpfer zum Verschwinden bringen und dafür den Erzähler einführen, der, anders als der Autor, glaubwürdig versichern kann, nichts zu erfinden, sondern wahrheitsgemäß zu berichten. Vorsätzlich verborgen hinter dem Erzähler, der nur Geschehenes vermittelt, kann der Autor die Souveränität des Erfindens und die Verantwortung dafür an das Schicksal abtreten, das sich somit in der unverstellten Wahrheit seines Verlaufs selber zur Darstellung bringt - unbeeinflußbar, wie das künftige Schicksal des Kindes der Agathe.

Wir sind am Ende unserer Überlegungen angelangt. Sie erlauben, ja verlangen - wie wir meinen -, daß die drei inhaltlichen Antinomien: Diener - Herr, Determinismus - Indeterminismus, Erzähler Leser unmittelbar voneinander abhängig sind.

Ihr inhaltliches Konvergieren führt zu einer strukturellen Kongruenz insofern, als sich die Struktur der sozialgeschichtlichen Dialektik von Herr und Knecht als maßgeblich für die Struktur des Fatalismusbegriffs und diese sich als bestimmend für die neue Problematik des Erzählens erweist. In der Komposition unseres Romans gelangt dieser Sachverhalt zur unwiderruflichen formalen Evidenz.

83 S. 777 ff. Der Autor treibt hier den Verzicht auf seine Allwissenheit so weit, daß er den Erzähler im Stich läßt. Der fragmentarische Abschluß bedeutet somit eine letzte Steigerung der antiromanesken Erzählhaltung. Ihm schließt sich ironisch die Schlußpointe an, in welcher offengelassen wird, ob die schöne Denise ihren Jacques zum Hahnrei werden läßt. Das Fragezeichen, das im Lazarillo de Tormes und im Gil Blas das Eheglück des Protagonisten in Zweifel zieht, wird am Ende von Jacques le Fataliste noch einmal zu jenem Fragezeichen, das die alleinige Antwort auf jede Zukunftserwartung darstellt. 